\title{
Spin Polarized Transport in an AC-Driven Quantum Curved Nanowire
}

\author{
Walid A. Zein, ${ }^{1}$ Nabil A. Ibrahim, ${ }^{2}$ and Adel H. Phillips ${ }^{1}$ \\ ${ }^{1}$ Faculty of Engineering, Ain-Shams University, Cairo 11517, Egypt \\ ${ }^{2}$ Higher Technological Institute, Ramadan Tenth City 44629, Egypt \\ Correspondence should be addressed to Adel H. Phillips, phillips_adel@yahoo.com
}

Received 17 September 2010; Accepted 20 April 2011

Academic Editor: Neil Sullivan

Copyright (C) 2011 Walid A. Zein et al. This is an open access article distributed under the Creative Commons Attribution License, which permits unrestricted use, distribution, and reproduction in any medium, provided the original work is properly cited.

\begin{abstract}
Using the effective-mass approximation method, and Floquet theory, we study the spin transport characteristics through a curved quantum nanowire. The spin polarization, $P$, and the tunneling magnetoresistance, TMR, are deduced under the effect of microwave and infrared radiations of wide range of frequencies. The results show an oscillatory behavior of both the spin polarization and the tunneling magnetoresistance. This is due to Fano-type resonance and the interplay between the strength of spin-orbit coupling and the photons in the subbands of the one-dimensional nanowire. The present results show that this investigation is very important, and the present device might be used to be a sensor for small strain in semiconductor nanostructures and photodetector.
\end{abstract}

\section{Introduction}

In the rapidly growing field of semiconductor spintronics, the spin degree of freedom is used for information processing $[1,2]$. Devices concepts have been proposed which offer lower power consumption and a higher degree of functionality [3]. Among the research area of spintronics, the spinorbit coupling (SOC) creates another way to manipulate spins by means of an electric field [4]. The Rashba spinorbit coupling effect [4] is found to be very pronounced in semiconductor heterostructure, for example, quantum dots, quantum wires, and quantum rings [5], and its strength can be controlled by gate voltage. The spin polarization in two-dimensional electron gas (2DEG) systems with spinorbit coupling (SOC) has been attracted extensive attention by many authors [6-9]. Spin-orbit coupling (SOC) has been investigated in parallel quantum wires $[10,11]$, where universal conductance fluctuations are suppressed. More recently, ballistic spin resonance due to an intrinsically oscillating spin-orbit field has been realized experimentally in a quantum wire [12]. The observation of the one-dimensional spin-orbit gap in quantum wires has also been reported in [13] and also recently in [14].

The aim of the present paper is to investigate the spin transport characteristics through a curved quantum nanow- ire under the effect of microwave (MW) and infrared (IR) radiations. The main difference between the straight nanowire and curved one is that the spin rotation is characterized by certain angles, as it will be shown below.

\section{The Model}

We will now derive an expression for both the spin polarization for spin injection current in the curved nanowire and the corresponding tunneling magnetoresistance (TMR) for a curved nanowire under the effect of induced photons of wide range of frequencies. This nanowire is connected to two metallic leads. The effective Hamiltonian for spin-injected electrons through one-dimensional nanowire can be written as three parts according the geometrical design of the curved nanowire as $[15,16]$

$$
\hat{H}_{1}=-\frac{\hbar^{2}}{2 m^{*}} \frac{\partial^{2}}{\partial S^{2}}-i \alpha \sigma_{b 1} \frac{\partial}{\partial S}+V_{d}+e V_{g}, \quad S \in(-\infty, 0),
$$

where $m^{*}$ is the effective mass of the electron, $V_{d}$ is the barrier height at the interface with the leads, $V_{g}$ is the gate voltage, and $S$ is the arc length along the curve. The second 
part of the curved nanowire including the Rashba spin-orbit coupling effect is given by

$$
\begin{array}{r}
\hat{H}_{2}=-\frac{\hbar^{2}}{2 m^{*} a^{2}} \frac{\partial^{2}}{\partial \theta^{2}}-\frac{\hbar^{2}}{8 m^{*} a^{2}}-\frac{i \alpha}{a}\left[\sigma_{b 2} \frac{\partial}{\partial \theta}-\frac{1}{2} \sigma_{t 2}\right], \\
\theta \in\left(0, \theta_{\omega}\right) .
\end{array}
$$

Also, the third part of the curved nanowire is

$$
\hat{H}_{3}=-\frac{\hbar^{2}}{2 m^{*}} \frac{\partial^{2}}{\partial S^{2}}-i \alpha \sigma_{b 3} \frac{\partial}{\partial S}+e V_{a c} \cos \omega t, \quad S \in\left(\theta_{\omega} a, \infty\right) .
$$

In (1), (2), and (3), the parameter $S$ represents the arc length along the curved part of the nanowire, $\theta$ is the polar angle, $\theta_{\omega}$ is the angle between two rectilinear parts of the nanowire and $V_{a c}$ is the amplitude of the induced photons with frequency $\omega$. The spin operators in (1), (2), and (3) are represented as follows [17]:

$$
\begin{gathered}
\sigma_{b 1}=-\sigma_{x}, \\
\sigma_{b 2}=-\sigma_{x} \cos \theta-\sigma_{y} \sin \theta, \\
\sigma_{b 3}=-\sigma_{x} \cos \theta_{\omega}-\sigma_{y} \sin \theta_{\omega}, \\
\sigma_{t 2}=-\sigma_{x} \sin \theta+\sigma_{y} \cos \theta .
\end{gathered}
$$

The energy spectrum and the unnormalized eigenstates for the two parts of the straight line of the nanowire are given by [18-20]

$$
\begin{gathered}
E^{\mu}=\frac{\hbar^{2} k^{2}}{2 m^{*}}-\mu \alpha k, \\
\Phi_{1, \lambda}^{\mu}=e^{i \lambda k s} \cdot \chi_{1}, \\
\Phi_{3, \lambda}^{\mu}=e^{i \lambda k s} \cdot \chi_{3},
\end{gathered}
$$

where the symbol $\mu$ corresponds to the spin up $\uparrow$ and spin down $\downarrow$, and $\lambda= \pm$ which corresponds to the direction of motion along the nanowire. The eigenstate function and the energy eigenvalue for the curved section of the nanowire are given as [18-20]

$$
\Phi_{2, \lambda}^{\mu}=e^{i \lambda n^{\prime} \theta} \cdot \chi_{2},
$$

where $n^{\prime}$ is the orbit quantum number

$$
E_{2, \lambda}^{\mu}=\Omega\left[\left(n^{\prime}+\frac{1}{2} \lambda\right)^{2}-\mu\left(n^{\prime}+\frac{1}{2} \lambda\right) \sqrt{1+\frac{\omega_{\text {Soc }}^{2}}{\Omega^{2}}}\right] .
$$

The spinors $\chi_{1}, \chi_{2}$, and $\chi_{3}$ in (6), (10), and (7) are given by

$$
\begin{gathered}
\chi_{1}^{ \pm}=\left(\begin{array}{c}
\frac{\sqrt{2}}{2} \\
\pm \frac{\sqrt{2}}{2}
\end{array}\right), \quad \begin{array}{l}
\text { [20]. Accordingly, therefore, the expressions for the tunnel- } \\
\text { ing probabilities corresponding to spin-up and spin-down } \\
\text { electrons, respectively, are given by }
\end{array} \\
\left|\Gamma_{\mu_{\mu \text { with photon }}}(E)\right|^{2}=\sum_{n} J_{n}^{2}\left(\frac{e V_{a c}}{\hbar \omega}\right) \times\left[\frac{8 A^{2} B^{2}\left(\cos ^{2} \xi+\cos \phi \cos (\phi-2 \xi) \cos \left[(1+2 \gamma) \theta+\sin ^{2}(\phi-\xi)\right]\right)}{(A+B)^{4}+(A-B)^{4}-2\left(A^{2}-B^{2}\right)^{2} \cos (2 \beta \theta)}\right] .
\end{gathered}
$$

$$
\begin{gathered}
\chi_{2}^{+}=\left(\begin{array}{c}
\sin \frac{\xi}{2} \\
e^{i \theta} \cos \frac{\xi}{2}
\end{array}\right), \\
\chi_{2}^{-}=\left(\begin{array}{c}
\cos \frac{\xi}{2} \\
-e^{i \theta} \sin \frac{\xi}{2}
\end{array}\right), \\
\chi_{3}^{ \pm}=\left(\begin{array}{c}
\frac{\sqrt{2}}{2} \\
\pm \frac{\sqrt{2}}{2} e^{i \theta} \omega
\end{array}\right) .
\end{gathered}
$$

In (8), we have the frequency associated with spin-orbit coupling $\omega_{\text {Soc }}$ and the parameter $\Omega$ which are defined as

$$
\omega_{\text {Soc }}=\frac{\alpha}{a \hbar}, \quad \Omega=\frac{\hbar^{2}}{2 m^{*} a^{2}},
$$

where $\alpha$ is the strength of the spin-orbit coupling and $a$ is the radius of curvature.

The eigenfunctions corresponding to the spin transport through a curved wire are given by [18-20]

$$
\begin{aligned}
\psi_{1}= & \sum_{n=-\infty}^{\infty} \sum_{\mu} J_{n}\left(\frac{e V_{a c}}{\hbar \omega}\right) \\
& \times\left[\cos (\phi) \Phi_{1,+}^{\mu}+\sin (\phi) \Phi_{1,+}^{\mu}+R_{\uparrow} \Phi_{1,-}^{\mu}+R_{\downarrow} \Phi_{1,-}^{\mu}\right], \\
\psi_{2}= & \sum_{\mu} \sum_{n=-\infty}^{\infty} J_{n}\left(\frac{e V_{a c}}{\hbar \omega}\right)\left[C_{1} \Phi_{2,+}^{\mu}+C_{2} \Phi_{2,-}^{\mu}\right], \\
\psi_{3}= & \sum_{\mu} \sum_{n=-\infty}^{\infty} J_{n}\left(\frac{e V_{a c}}{\hbar \omega}\right)\left[\Gamma_{\uparrow} \Phi_{3,+}+\Gamma_{\downarrow} \Phi_{3,-}\right],
\end{aligned}
$$

where $J_{n}\left(e V_{a c} / \hbar \omega\right)$ is the $n$th order Bessel function. The solutions of (11) must be generated by the presence of different subbands, $n$, in a quantum nanowire, which come with phase factor $\exp (-i n \omega t)$, where $\omega$ is the frequency of the induced photons. Now, the tunneling probability $\left|\Gamma_{\mu \text { with photon }}(E)\right|^{2}$ could be obtained by applying Griffith boundary conditions 
And that for spin-down as

$$
\left|\Gamma_{\downarrow_{\mu \text { with photon }}}(E)\right|^{2}=\sum_{n} J_{n}^{2}\left(\frac{e V_{a c}}{\hbar \omega}\right)\left[\frac{8 A^{2} B^{2}\left(\cos ^{2}(\phi-\xi)-\cos \phi \cos (\phi-2 \xi) \cos [(1+2 \gamma) \theta]+\sin ^{2} \xi\right)}{(A+B)^{4}+(A-B)^{4}-2\left(A^{2}-B^{2}\right)^{2} \cos (2 \beta \theta)}\right] .
$$

where the parameters, in (12) and (13), $A, B, \beta$, and $\gamma$, are expressed as

$$
\begin{gathered}
A=\sqrt{\left(\frac{\alpha}{\hbar}\right)^{2}+\frac{2\left(E_{F}+V_{d}+e V_{g}+n \hbar \omega\right)}{m^{*}},} \\
B=\sqrt{\left(\frac{\alpha}{\hbar}\right)^{2}+\frac{2\left(E_{F}+(\Omega / 4)+V_{d}+e V_{g}+n \hbar \omega\right)}{m^{*}},} \\
\beta=\frac{B \hbar}{2 a \Omega}, \quad \gamma=-\frac{1}{2}+\frac{1}{2} \sqrt{1+\frac{\omega_{\text {Soc }}^{2}}{\Omega^{2}}}, \\
\tan \phi=-\frac{\omega_{\text {Soc }}}{\Omega} .
\end{gathered}
$$

So, the spin polarization of the tunneled electrons [21] is

$$
P=\frac{\left|\Gamma_{t_{\mu \text { with photon }}}(E)\right|^{2}-\left|\Gamma_{\downarrow_{\mu \text { with photon }}}(E)\right|^{2}}{\left|\Gamma_{t_{\mu \text { with photon }}}(E)\right|^{2}+\left|\Gamma_{\downarrow_{\mu \text { with photon }}}(E)\right|^{2}}
$$

In order to investigate the spin injection tunneling through the curved nanowire, we could calculate the tunneling magnetoresistance (TMR) which is related to the spin polarization (15) as [21-23]:

$$
\mathrm{TMR}=\frac{P^{2}}{1-P^{2}+\Gamma_{S}},
$$

where $\Gamma_{S}$ is the relaxation parameter and is given by [21-23]:

$$
\Gamma_{S}=\frac{e^{2} N(0) R_{T} A \ell}{\tau_{S}},
$$

where $N(0)$ is the normal-state density of electrons calculated for both spin-up and spin-down distribution function $f_{\sigma}(E)$, which is expressed as [21-23]

$$
f_{\sigma}(E) \cong f_{0}(E)-\left(\frac{\partial f_{0}}{\partial E}\right) \cdot \mu \delta f
$$

where $\delta f$ is the shift of the chemical potential, $\tau_{S}$ is the spin relaxation time, $A$ is the cross-sectional area of the nanowire, and $R_{T}$ is the resistance at the interface of the tunnel junction.

\section{Result and Discussion}

The nanowire is the semiconductor heterostructure InAsInGaAs with characteristic values $m^{*}=0.023 m_{e}, E_{F}=$ $11.13 \mathrm{meV},|\theta| \leq \pi[14,16,17]$. The features of our present results are the following.

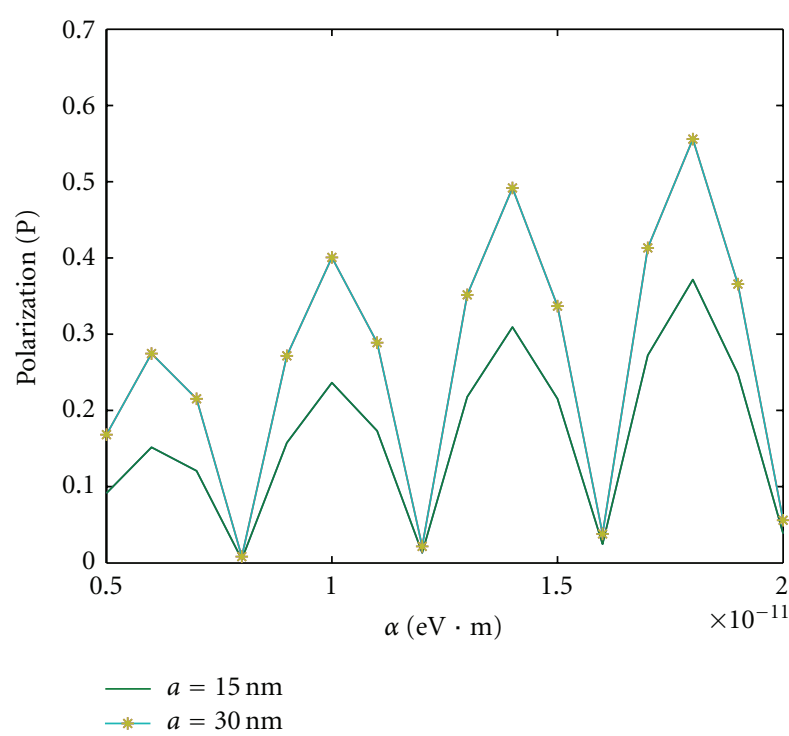

FIgURE 1: The variation of the spin polarization with the strength of the spin-orbit coupling, $\alpha$, at different values of the radius of curvature of the nanowire.

(i) Figure 1 shows the variation of the spin polarization with the strength of the spin-orbit coupling, $\alpha$, at different values of the radius of the curvature of the nanowire. The results show periodic oscillations of the polarization. Also, the peak heights vary in a quantized form for the two values of a (radius of curvature).

(ii) Figure 2 shows the variation of the tunneling magnetoresistance (TMR) with the strength of the spin-orbit coupling, $\alpha$. As in Figure 1, periodic oscillations of the tunneling magnetoresistance (TMR) are observed. Also, peak heights vary in a quantized form for the two values of the radius of the curvature for nanowire. Such results show that the spin transport through curved nanowire is very sensitive to the geometrical shape of the nanowire. The strength of the spin-orbit coupling, $\alpha$, can be controlled by the gate voltage, the energy of the induced photons and the geometrical shape. Such results are found to be concordant with those in the literature $[7,11,14]$.

(iii) Figure 3 , shows the variation of polarization with the photon energy at different values of the strength of the spinorbit coupling, $\alpha$. An oscillatory behavior of the polarization is observed. This is due to Fano-type resonance [24-27].

(iv) Figure 4 shows the variations of the tunneling magnetoresistance (TMR) with the photon energy at different values of the strength of SOC. Oscillations are observed as 


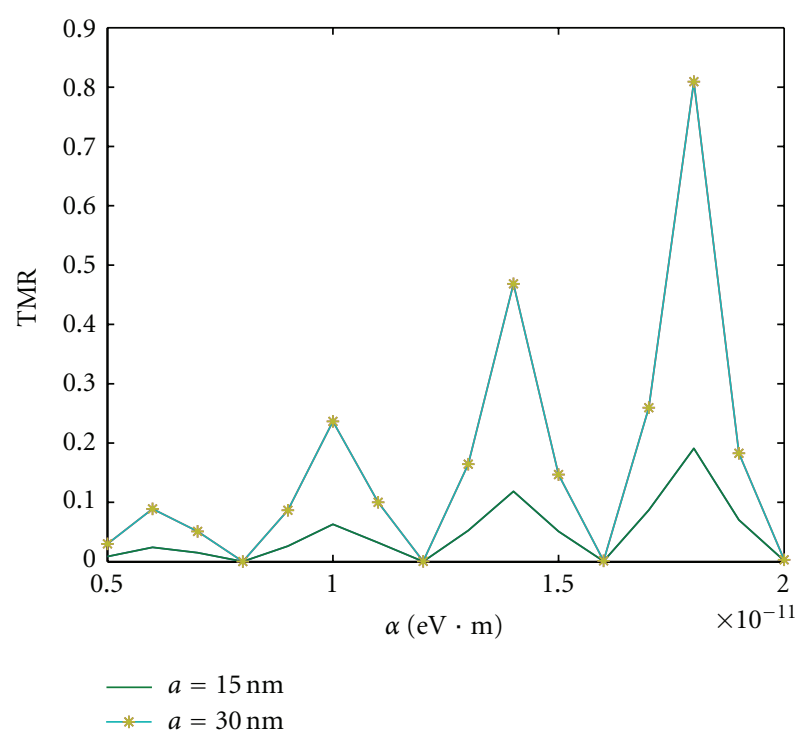

FIGURE 2: The variation of the tunneling magnetoresistance (TMR) with the strength of the spin-orbit coupling, $\alpha$, at different values of the radius of curvature of the nanowire.

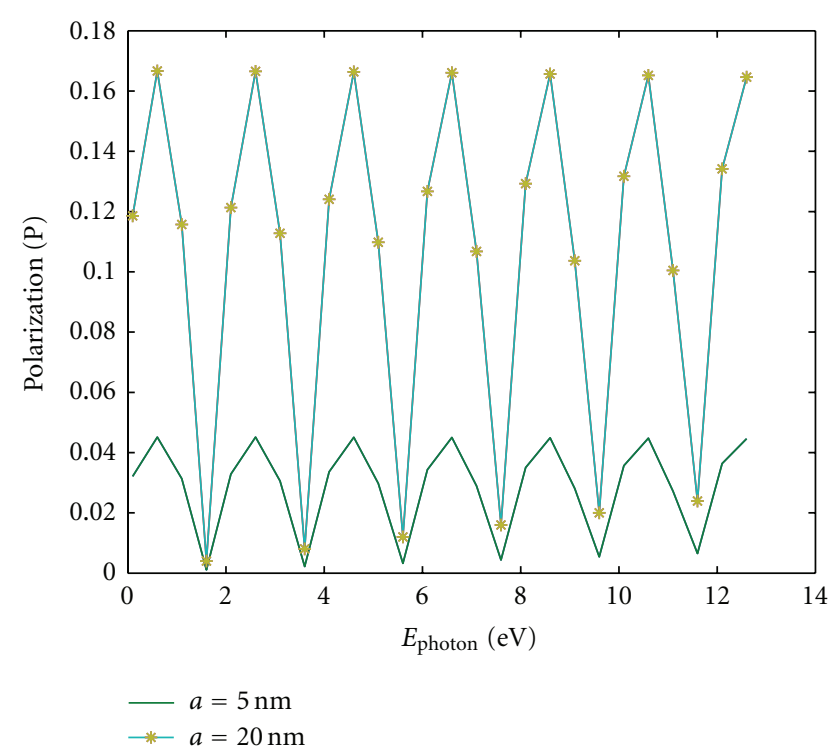

Figure 3: The variation of the spin polarization with the photon energy at different values of the radius of curvature of the nanowire.

in the case of the spin polarization (Figure 3). These results show a good concordant with those in the literature [24-27]. These results show that the location and line shape of Fanotype resonance can be controlled by both the frequency of the induced photons and the strength of the spin-orbit coupling.

We can conclude that the present investigation is very important for devising a mesoscopic nanowire with controllable curvature. By this device, we can determine very minute strain in semiconductor heterostructure solids [28]. Also, this nanowire can be used as a photodetector [29].

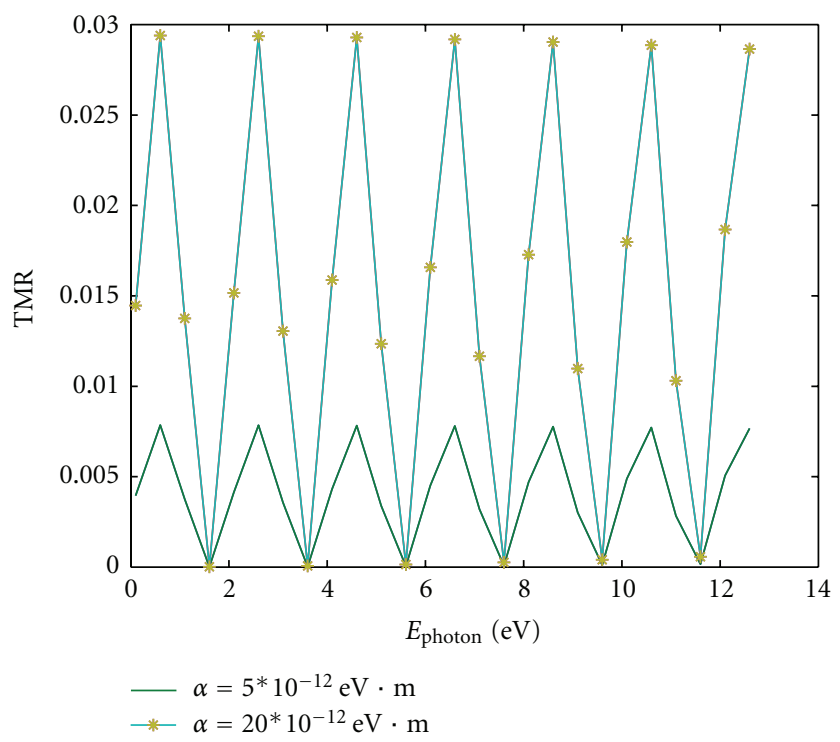

FIGURE 4: The variation of the tunneling magnetoresistance (TMR) with the photon energy at different values of the strength of SOC.

\section{References}

[1] P. Ball, "Meet the spin doctors...," Nature, vol. 404, no. 6781, pp. 918-920, 2000.

[2] S. A. Wolf, D. D. Awschalom, R. A. Buhrman et al., "Spintronics: a spin-based electronics vision for the future," Science, vol. 294, no. 5546, pp. 1488-1495, 2001.

[3] I. Žutić, J. Fabian, and S. D. Sarma, "Spintronics: fundamentals and applications," Reviews of Modern Physics, vol. 76, no. 2, pp. 323-410, 2004.

[4] E. I. Rashba, "Electron spin operation by electric fields: spin dynamics and spin injection," Physica E, vol. 20, no. 3-4, pp. 189-195, 2004.

[5] R. Winkler, Spin-Orbit Coupling Effect in Two Dimensional Electron and Hole Systems, Springer, New York, NY, USA, 2003.

[6] Z. Li and Z. Yang, "Effects of extended and localized states on spin Hall polarization in ballistic Rashba structures," Physical Review B, vol. 77, no. 20, Article ID 205322, 2008.

[7] S. Bellucci and P. Onorato, "Quantum wires as logic operators: XNOR and NOR gate response in a ballistic interferometer," Physical Review B, vol. 81, no. 16, Article ID 165427, 2010.

[8] Q. Wang and L. Sheng, "Current induced local spin polarization due to the spin-orbit coupling in a two dimensional narrow strip," International Journal of Modern Physics B, vol. 19, no. 27, pp. 4135-4142, 2005.

[9] M. Lee, M. O. Hachiya, E. Bernardes, J. C. Egues, and D. Loss, "Spin Hall effect due to intersubband-induced spin-orbit interaction in symmetric quantum wells," Physical Review B, vol. 80, no. 15, Article ID 155314, 2009.

[10] V. A. Guzenko, A. Bringer, J. Knobbe, H. Hardtdegen, and T. Schäpers, "Rashba effect in GaInAs/InP quantum wire structures," Applied Physics A, vol. 87, no. 3, pp. 577-584, 2007.

[11] Th. Schäpers, V. A. Guzenko, A. Bringer, M. Akabori, M. Hagedorn, and H. Hardtdegen, "Spin-orbit coupling in GaxIn1$\mathrm{xAs} / \mathrm{InP}$ two-dimensional electron gases and quantum wire structures," Semiconductor Science and Technology, vol. 24, no. 6, Article ID 064001, 2009.

[12] S. M. Frolov, S. Lüscher, W. Yu, Y. Ren, J. A. Folk, and W. 
Wegscheider, "Ballistic spin resonance," Nature, vol. 458, no. 7240, pp. 868-871, 2009.

[13] A. Tsukernik, A. Palevski, V. J. Goldman, S. Luryi, E. Kapon, and A. Rudra, "Quantum magnetotransport in periodic Vgrooved heterojunctions," Physical Review B, vol. 63, no. 15, Article ID 153315, 4 pages, 2001.

[14] C. H. L. Quay, T. L. Hughes, J. A. Sulpizio et al., "Observation of a one-dimensional spin-orbit gap in a quantum wire," Nature Physics, vol. 6, no. 5, pp. 336-339, 2010.

[15] R. C. T. Da Costa, "Constraints in quantum mechanics," Physical Review A, vol. 25, no. 6, pp. 2893-2900, 1982.

[16] B. K. Nikolić, L. P. Zĝrbo, and S. Welack, "Transverse spinorbit force in the spin Hall effect in ballistic semiconductor wires," Physical Review B, vol. 72, no. 7, Article ID 075335, 6 pages, 2005.

[17] X. Fu, W. Liao, and G. Zhou, "Spin accumulation in a quantum wire with Rashba Spin-Orbit coupling," Advanced in Condensed Matter Physics, vol. 2008, Article ID 152731, 5 pages, 2008.

[18] W. A. Zein, A. H. Phillips, and O. A. Omar, "Quantum spin transport in mesoscopic interferometer," Progress in Physics, vol. 4, pp. 18-21, 2007.

[19] W. A. Zein, A. H. Phillips, and O. A. Omar, "Spin-coherent transport in mesoscopic interference device," NANO, vol. 2, no. 6, pp. 389-392, 2007.

[20] W. A. Zein, N. A. Ibrahim, and A. H. Phillips, "Spindependent transport through Aharonov-Casher ring irradiated by an electromagnetic field," Progress in Physics, vol. 4, pp. 78-80, 2010.

[21] W. A. Zein, A. H. Phillips, and O. A. Omar, "Spin transport in mesoscopic superconducting-ferromagnetic hybrid conductor," Progress in Physics, vol. 1, pp. 42-46, 2008.

[22] A. A. Awad Alla, A. H. Aly, and A. H. Phillips, "Electron spin dynamics through ferromagnetic quantum point contact," International Journal of Nanoscience, vol. 6, no. 1, pp. 41-44, 2007.

[23] S. Takahashi, T. Yamashita, H. Imamura, and S. Maekawa, "Spin-relaxation and magnetoresistance in FM/SC/FM tunnel junctions," Journal of Magnetism and Magnetic Materials, vol. 240, no. 1-3, pp. 100-102, 2002.

[24] F. M. Souza, "Spin-dependent ringing and beats in a quantum dot system," Physical Review B, vol. 76, no. 20, Article ID 205315, 2007.

[25] K. Kobayashi, H. Aikawa, S. Katsumoto, and Y. Iye, “Tuning of the Fano effect through a quantum dot in an Aharonov-Bohm interferometer," Physical Review Letters, vol. 88, no. 25, Article ID 256806, 4 pages, 2002.

[26] K. Kobayashi, H. Aikawa, A. Sano, S. Katsumoto, and Y. Iye, "Fano resonance in a quantum wire with a side-coupled quantum dot," Physical Review B, vol. 70, no. 3, Article ID 035319, 2004.

[27] A. T. Tilke, F. C. Simmel, H. Lorenz, R. H. Blick, and J. P. Kotthaus, "Quantum interference in a one-dimensional silicon nanowire," Physical Review B, vol. 68, no. 7, Article ID 075311, 6 pages, 2003.

[28] S. S. Kwon, W. K. Hong, G. Jo et al., "Piezoelectric effect on the electronic transport characteristics of $\mathrm{ZnO}$ nanowire field-effect transistors on bent flexible substrates," Advanced Materials, vol. 20, no. 23, pp. 4557-4562, 2008.

[29] W. Wu, S. Bai, N. Cui et al., "Increasing UV photon response of $\mathrm{ZnO}$ sensor with nanowire array," Science of Advanced Materials, vol. 2, pp. 402-406, 2010. 

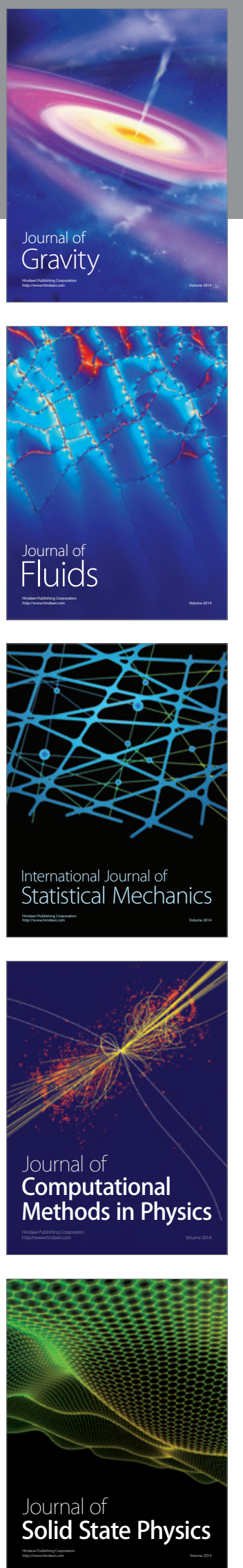

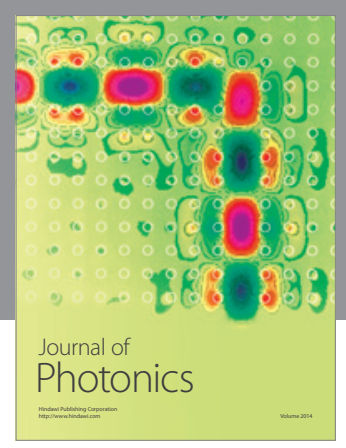

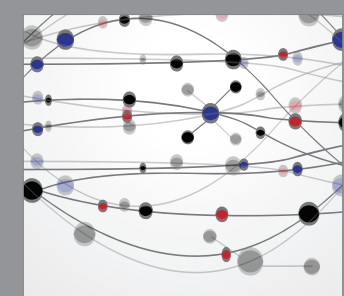

The Scientific World Journal
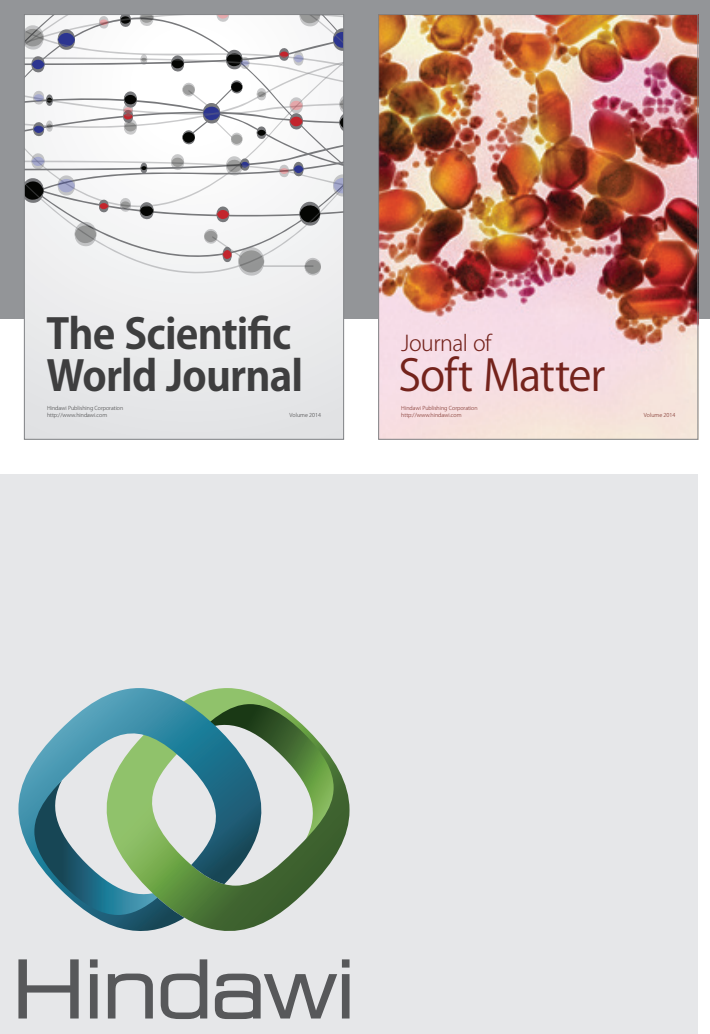

Submit your manuscripts at

http://www.hindawi.com
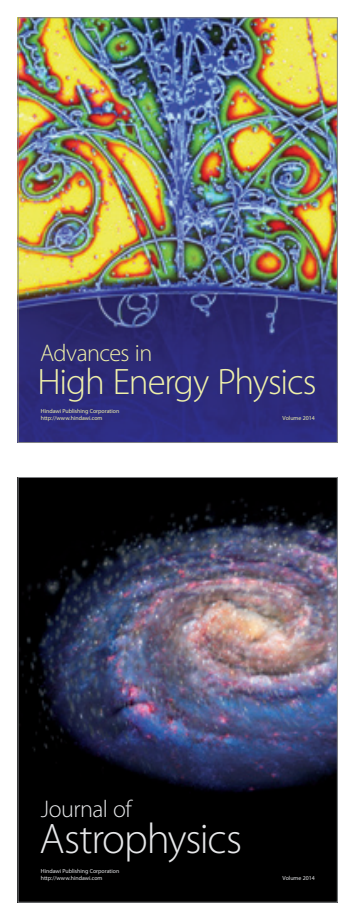
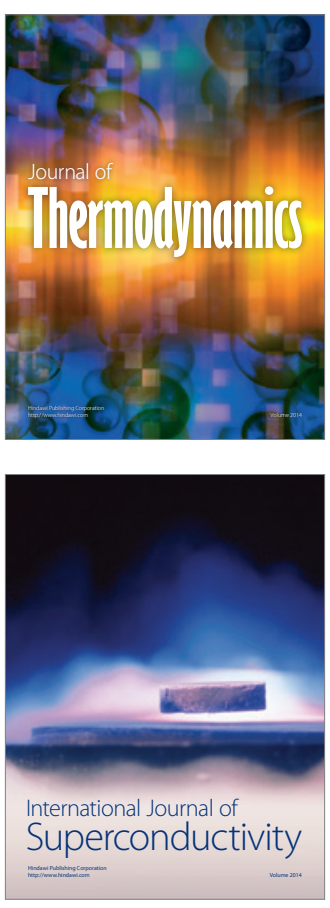
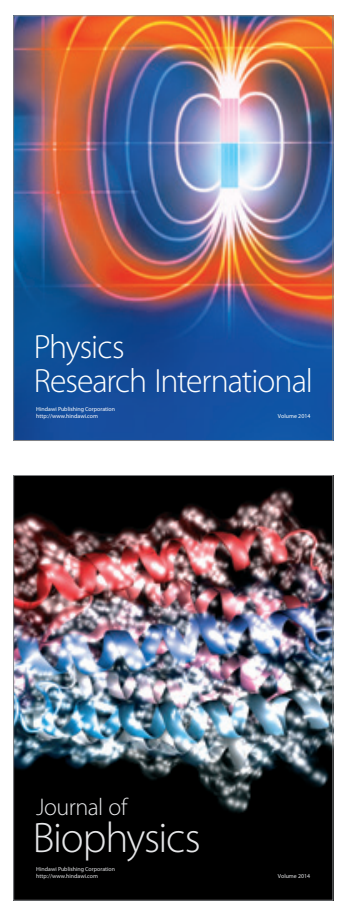
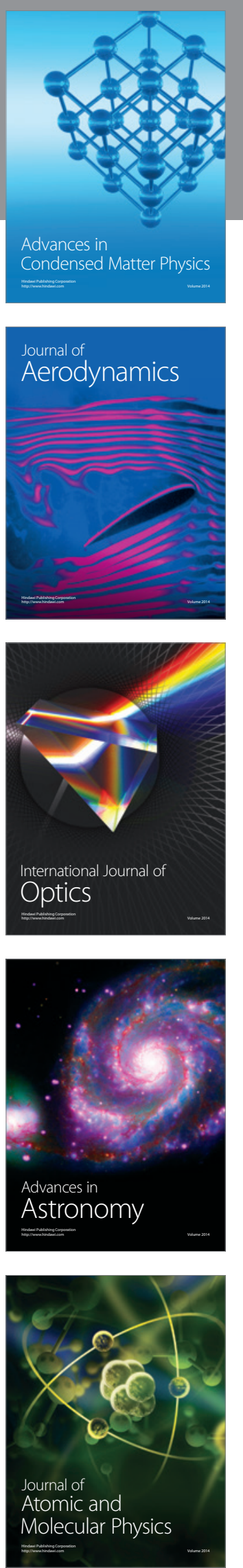